\title{
Introduction of Handover Manager for Better Handover Latency and Handover Throughput during Leo Satellite Handover
}

\author{
Debabrata sarddar (PhD \\ Scholar) \\ Department of E.T.C.E. \\ Jadavpur University \\ Suman Kumar Sikdar \\ M. Tech in C.S.E \\ Jadavpur University \\ Shabnam \\ Bandyopadhyay \\ Department of C.S.E \\ Jadavpur University
}

\author{
Dipsikha Ganguly \\ Department of E.T.C.E. \\ B.P.Poddar I.M.T.,WBUT
}

\author{
Sougata Chakraborty \\ M.Tech in C.S.E \\ Jadavpur University
}
Kallyan Kumar Das
M. Tech in C.S.E
Jadavpur University

\author{
Soumya Das \\ Department of E.T.C.E \\ Bengal Institute of tec.WBUT \\ Kunal Hui \\ M. Tech in C.S.E \\ Haldia Institute of Technology \\ Mrinal Kanti Naskar \\ Department of E.T.C.E \\ Jatavpur University
}

\begin{abstract}
LEO satellite has an important role in global communication system. They have advantages like low power requirement and lower end-to-end delay, efficient frequency spectrum utilization between satellites and spotbeams over MEO and GEO satellites. So in future they can be used as a replacement of modern terrestrial wireless networks. There are a lot of handover techniques for LEO satellites like seamless handover (SeaHO-LEO) ,PatHO-LEO .In our proposed work we have suggested a new handover technique for SeaHOLEO by introducing a Handover Manager (HM) during the handover process and by simulation we have also shown that it a better approach by comparing it with other existing handover techniques as it reduces the handover latency, propagation delay, call blocking probability more than any other technique.
\end{abstract}

\section{Keywords}

Handover latency, LEO, Mobile Node (MN),Handover Manager (HM)

\section{INTRODUCTION}

Satellite communication networks are utilized to co exist with terrestrial networks to provide global coverage to a heterogeneously distributed over population,. A LEO satellite takes about 100 minutes to orbit the earth, which means that a single satellite is "in view" of ground equipment for only a few minutes [1]. As a consequence, if a transmission takes more than the short time period than any one satellite.

\section{Handover:}

In cellular telecommunications, the term handover or handoff refers to the process of transferring an ongoing call or data session from one channel connected to the core network to another. In satellite communications it is the process of transferring satellite control responsibility from one earth station to another without loss or interruption of service.

Types of handover
A Hard Handover is one in which the channel in the source cell is released and only then the channel in the target cell is engaged. Thus the connection to the source is broken before or 'as' the connection to the target is made-for this reason such handovers are also known as break-before-make. Hard handovers are intended to be instantaneous in order to minimize the disruption to the call. When the mobile is between base stations, then the mobile can switch with any of the base stations, so the base stations bounce the link with the mobile back and forth. A Soft Handover is one in which the channel in the source cell is retained and used for a while in parallel with the channel in the target cell. In this case the connection to the target is established before the connection to the source is broken, hence this handover is called makebefore-break. The interval, during which the two connections are used in parallel, may be brief or substantial. Soft handovers may involve using connections to more than two cells: connections to three, four or more cells can be maintained by one phone at the same time. The latter is more advantageous, and when such combining is performed both in the downlink (forward link) and the uplink (reverse link) the handover is termed as softer. Softer handovers are possible when the cells involved in the handovers have a single cell site. A LEO satellite system must hand over between satellites to complete the transmission. In general, this can be accomplished by constantly relaying signals between the satellite and various ground stations, or by communicating between the satellites themselves using "inter-satellite links"(ISLs) [1], [2]. LEO satellites are also designed to have more than one satellite in view from any spot on the earth at any given time, minimizing the possibility that the network will loose the transmission. Due to the fast-flying satellites, LEO systems must incorporate complicated tracking and switching equipment to maintain consistent service coverage. In this paper, we focus on the handover management of satellite networks, which is a crucial design problem for supporting mobile communication services in the co-existing terrestrial and LEO satellite networks. One of the proposed 
models for handover management in satellite networks is mobile IP (MIP) [3]. When a mobile host moves from one point of attachment to another it enables a TCP connection to remain alive and to continue receiving packets. Although MIP is a widely used approach applied to satellite networks, it has some important drawbacks including high handover latency and high packet loss[4][5].

In our paper we have proposed an idea of reducing binding updates, handover latency and packet loss during handover. Our paper is structured as follows:

In the first section we have given a brief introduction related to LEO satellite and handover mechanisms. In section 2 we have discussed the related work regarding MIP network. In next section we have proposed our idea of introducing a location manager .In section $4 \& 5$ we have shown the simulation results and the conclusion and the future work regarding this paper.

\section{RELATED WORK}

The most widely used protocol for handover in satellite is MIP [7]. It is proposed by The Internet engineering task force (IETF) to handle mobility of internet hosts for mobile data communications. MIP is based over the concept of Home Agent (HA) and Foreign Agent (FA) for delivering of packets from one $\mathrm{MN}$ to $\mathrm{CN}$.

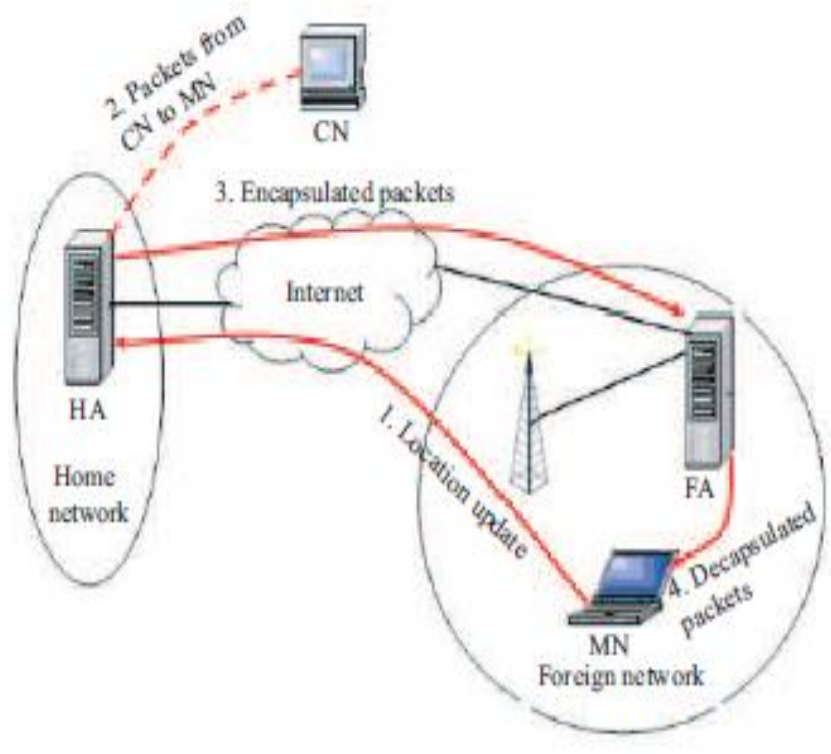

Figure 1: Handover Flow of Mobile IP

It is basically completed by four steps.

i) When handover begins $\mathrm{MN}$ registers itself in FA and waits for allocation of channels in FA and updates its location in HA directory.

ii) The packets are sent to HA and HA encapsulate it.

iii) Encapsulated packets are sent to The FA.

iv) FA decapsulate those packets and sent it to MN.

The main drawback of this protocol is

a. High handover latency

b. High packet lost rate

c. Insufficient routing path

d. Conflicts with network security solution

Another method is Seamless handover management scheme (SEAHO-LEO) [8],[9] proposed by Aysegul et al in 2006.
It reduces packet loss and handover latency. It is describes as follows

A. Calculate a new IP

B. Send handover preparation request to current satellite

C. Start to use new IP to send data packets

D. CN starts to use new satellite

SEAHO-LEO provides efficient utilization of network bandwidth because of the absence of tunneling and also does not need any change in existing internet infrastructure.

The main disadvantage of this process is high messaging traffic.

\section{SeaHO-LEO Handover}

In SeaHO-LEO CN does not move, so its location is fixed. The Fig. illustrates the integration of terrestrial IP network and LEO satellite networks.

Handover in SeaHO-LEO: The mean number of available satellites for a given $\mathrm{MN}$ is at least two for latitudes less than 60 degrees [16]. This means that MN is within the footprint of two satellites most of the time, which makes the model effective for handover management of satellites to reduce packet loss and handover latency. The handover procedure is as follows:

1) Calculate a new IP: The handover preparation procedure generally begins when the MN moves into the overlapping coverage area of two adjacent satellites' footprints. As illustrated in Fig. 2, when the $\mathrm{MN}$ receives the agent advertisement

(AA) from the new satellite (i.e., $\mathrm{SN}$ ), it will initiate the steps of obtaining a new IP address.

MN measures the received signal strength first and then determines the need for handover when the signal strength of the current radio link deteriorates below a certain threshold while still allowing communications. If there are more than one satellites for handover, MN should choose the most favorable one according to the connections QoS parameters.

After determining the favorable satellite, it starts to obtain a new IP for new satellite. Obtaining new IP address can be performed with a dynamic host configuration protocol (DHCP) [17], dynamic host configuration protocol for IPv6 (DHCPv6) [18], or IPv6 stateless address auto-configuration (SAA) [19]. The main difference of these methods is the generation of IP addresses either by a server (DHCP/DHCPv6) or by the MN itself (IPv6 SAA). Since the IPv6 SAA significantly reduces the required signaling time, it is recommended or the proposed handover protocol. new IP generation step, $\mathrm{MN}$ only requires the address to be unique and routable.

2) Send handover preparation request to current satellite: After MN computes a new IP address, it sends a handover preparation request (i.e., HP REQUEST) to the current satellite (SC). This message includes new IP of MN, and the IP of SN. After SC receives HP REQUEST, it replies to MN by sending a handover preparation response (i.e., HP RESPONSE) message to indicate the success of receiving the new IP. At that time, SC also starts buffering all inbound packets on SN during the handover. Thus, all the inbound packets coming to the $\mathrm{SC}$ for $\mathrm{MN}$ will also be sent directly to the next satellite SN by using ISL between SC and SN. The buffering mechanism creates a virtual path between $\mathrm{CN}$ and the new satellite $\mathrm{SN}$. This buffering procedure is an intelligent one in which the current satellite does not send the packets whose acknowledgements from MN delivered by SC. Therefore, SN receives only packets that are not received by $\mathrm{MN}$. 
3) use of new IP to send data packets: When MN moves further into the footprint of the new satellite $\mathrm{SN}$, data path between $\mathrm{MN}$ and $\mathrm{SN}$ becomes increasingly more reliable than data path between MN and current satellite SC. At that time, MN may want to start to redirect data traffic to the new IP to increase the possibility of successful delivery of data packets to the MN. Therefore, MN sends a handover ready request (i.e., HR REQUEST) to current satellite SC to say that new IP is available for data transmission. When SC receives HR REQUEST message, new IP is sent to $\mathrm{CN}$ by the message CONN ACTIVATE. When CN receives the new IP, it replies to current satellite with an acknowledgement indicating the success of obtaining the new IP. It also stops buffering the inbound packets on next satellite SN since the new IP is ready to receive new packets. At that point, SC sends a CONN ACTIVATE message to SN to make connection establishment complete After SC receives HR REQUEST message.

When SN receives this message, it understands that MN will communicate with $\mathrm{CN}$ via SN. In other words, new IP is ready for the communication. Therefore, the final message causes the next satellite $\mathrm{SN}$ to forward all the buffered packets to the MN. As soon as the new IP becomes ready to receive packets, SN sends all the buffered packets to $\mathrm{MN}$ and starts communication with MN. Moreover, SN sends a $\mathrm{HO}$ RELEASE message to SC to complete disassociation and release of radio resources at the old satellite.

4) CN starts to use new satellite: After the new IP of the MN is received, it sends the next data packets to $\mathrm{MN}$ via new satellite SN. The SeaHO-LEO provides efficient utilization of network bandwidth because of the absence of tunneling, and also does not need any change in the existing internet infrastructure. SeaHO-LEO uses the efficiency of ISL. Therefore, our model is very effective to provide continuous communication between a $\mathrm{CN}$ and a MN whose mobility is random.

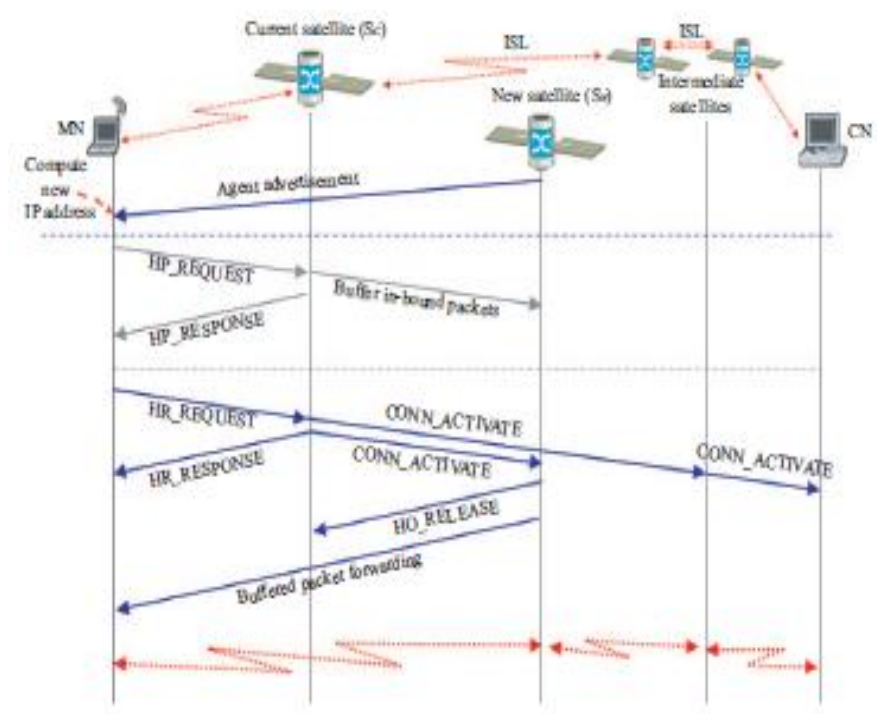

Fig 2.SeaHO-LEO HANDOVER

\section{PROPOSED WORK}

In our proposed work we have worked on the handover in SeaHO-LEO satellites. Here we have worked on the virtual environment of Aysegul et al. Here we have discussed about a new handover scheme of SEAHO-LEO by modifying the old one discussed in[21][22].

In MIP system each subnet has to locate a location management component (HA) which is a very difficult task while the number of users and location management entities increase. So, SeaHO-LEO system is proposed to reduce the complexity and the mobility management and the maintenance cost. The research results in [] show that the mean number of available satellites for a given $\mathrm{MN}$ is at least two for latitudes less than 60 degrees. This means that $\mathrm{MN}$ is within the footprint of two satellites most of the time, which makes our model effective for handover management of satellites to reduce packet loss and handover latency.

In our proposed work we have just modified the procedure of handover of SeaHO-LEO, here we have introduced a new Handover Manager(HM) which will reduce the binding updates and handover latency as it shows that there is no need to send and receive handover preparation request prior to handover. so, the process of the handover of the SeaHO-LEO is just as follows:

1) First initiates the process by searching for the first IP or the new IP when the MN moves into the overlapping area of two adjacent satellite footprints, determining the signal strength and the QOS parameter choose the ideal one and register it.

Find out the next IP where the packets need to be forwarded by measuring the signal strength of the satellites while the satellite is moving in a network .The IP address of two adjacent satellite is stored in handover manager for the fraction of second until the next IP arises and by comparing the signal strength it will keep the IP of maximum signal strength.

2) Now when the handover is needed there is no need to send handover preparation or the handover response request as the IP is already stored in the handover manager(HM).

3) Handover will be initiated.

4) The data packets will be forwarded to the new ip stored in HM .

5) During the forwarding of packets HM will keep searching for the IP and when the first data forwarding will be completed it will start sending to the next IP stored.

6) Thus the process continues. 


\section{Flowchart:}

The flowchart of the proposed handover has been shown in the fig 3 .

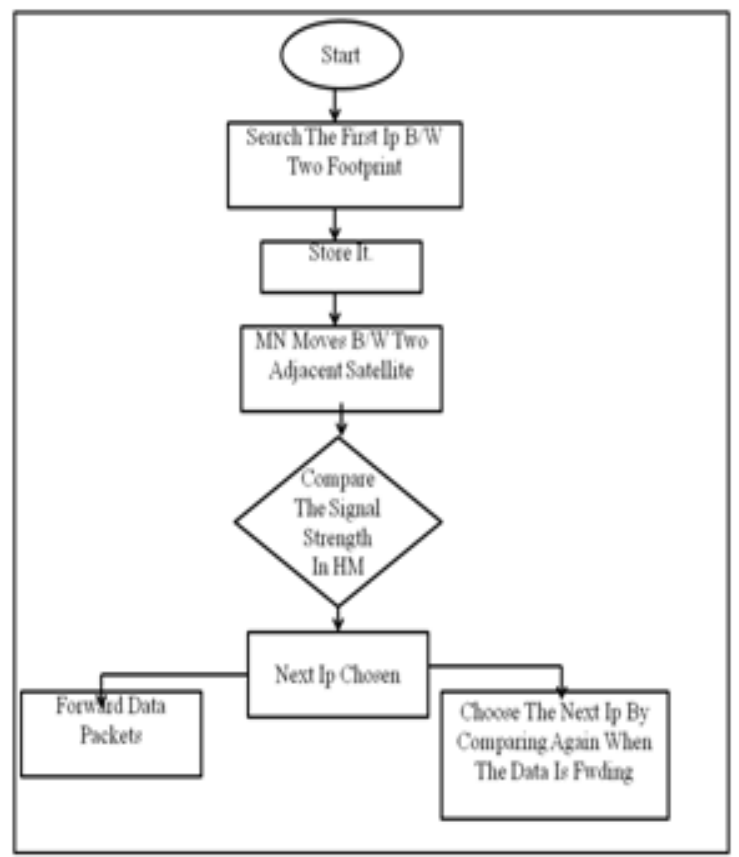

Fig 3 Flow chart of this proposed method:

It will reduce the binding updates as well as handoff latency as there is no need to register for the handoff preparation request and handoff response request. Introduction of HM will both the thing itself. Also there is no extra searching time for the new IP. It will find it while the $\mathrm{MN}$ will move to different ips by comparing the signal strength.

Now by simulation we have shown our proposed is better than the existing one and also the Mobile IP Network.

\section{SIMULATION RESULT:}

In order to evaluate the performance HANDOVER MANAGER with SeaHO-LEO, we compared it to MIP \& SeaHO-LEO scheme. Each algorithm is evaluated by analyzing the Handoff delay, Forced call termination probability \& Handover latency. The simulation results have been shown using the MATLAB 7.8 in a designed virtual environment.

In figure 4 we compare the Handover throughput for MIP, SeaHO-LEO \& Handover Manager i.e. the proposed SEAHOLEO handover throughput during a handover process. In mobile IP, due to the tunneling between HA and FA, throughput of the channel between MN1/CN and MN2/MN converges to zero during handover. When the handover model is completed, the throughput reaches a reasonable value. The throughput of SeaHO-LEO is better than MIP during handover as it does not reach to zero. In BMBHO the throughput is higher than SeaHO-LEO because the handover takes very less time and the packets during handover is sent by the old link.

In MIP the MN has to search for a new satellite \& then analyze them. For SeaHO-LEO the handoff delay is closer to MIP but in our work as everything is kept within BM \& BM just have to run a simple algorithm so the handoff delay is very much less.

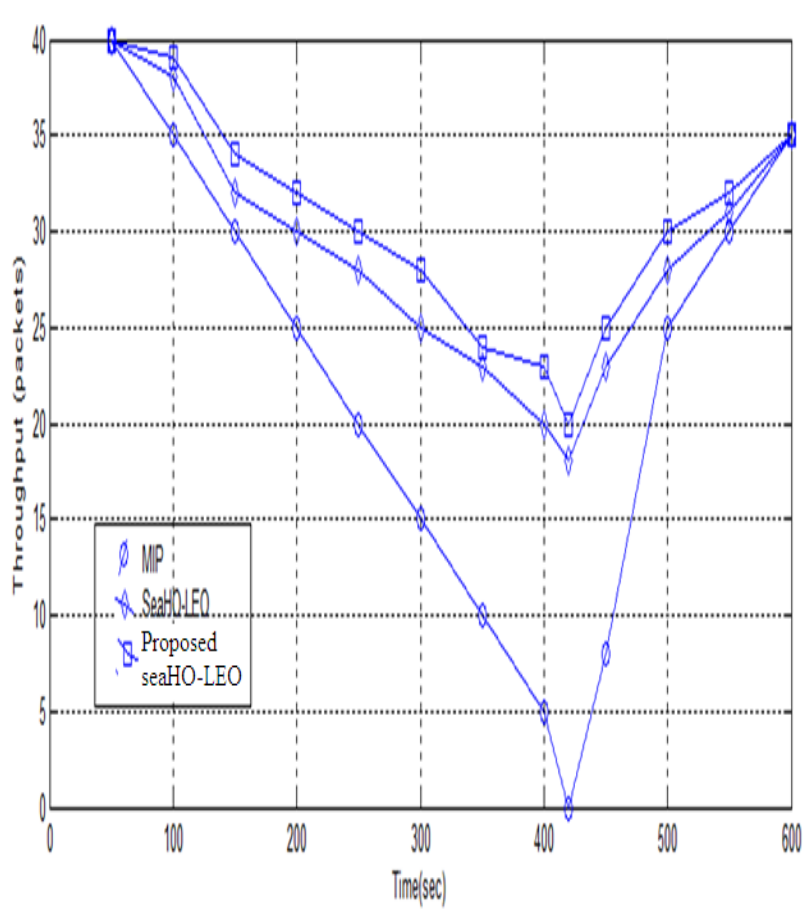

Figure 4: Simulation results of MN's handover throughput

In figure 5 we compare the Forced call termination probability of MIP \& SeaHO-LEO with the proposed SeaHO-LEO. Among this three handover management models, HANDOVER MANAGER i.e. our new proposed seaHO-LEO has the lowest Forced call termination probability. In MIP the $\mathrm{MN}$ has to wait for the channel allocation \& if it did not get a free channel within the handoff time the call is being terminated. In SeaHO-LEO the MN has to wait for the agent advertisement from a new satellite. If it did not get it within handoff time the call is being terminated. But in HM there is no need to wait as it initatializes the next call earlier during the first packet forwarding by comparing the adjacent satellite's signal strength .Hence the call blocking probability is very less.

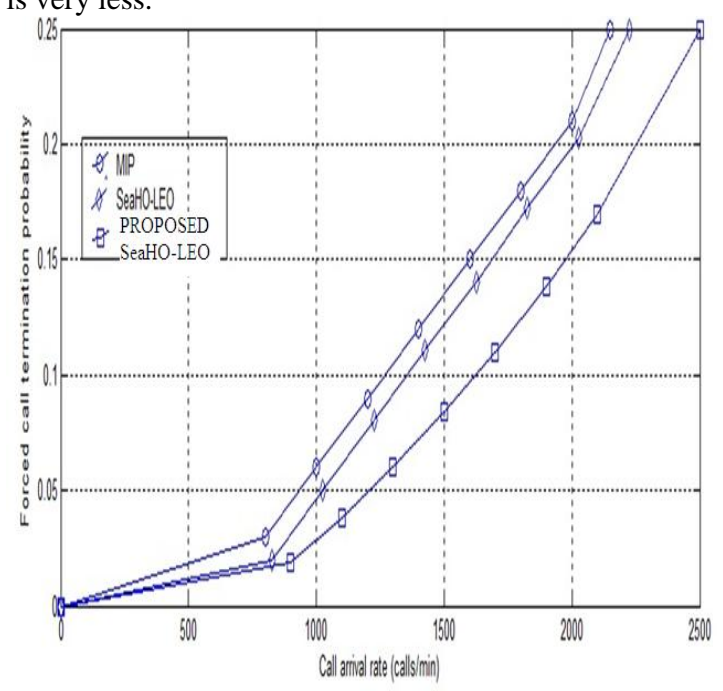

Figure 5: Forced call termination probability of a handover call. 
In figure 6 we have shown the average handoff latency. Handoff latency affects the service quality of real time applications of mobile users. It is dependent on the time taken to establish a new path segment between $\mathrm{MN}$ and new satellite. Basically it is the time interval between the last data segment received through the old path and the first data segment received through the new path from $\mathrm{CN} / \mathrm{MN} 2$ to MN/MN1.

In MIP, the handover latency is immense because $\mathrm{MN}$ has to send location update message to it's HA to associate its home address and CoA. This binding update process is time consuming operation. MIP is incapable of receiving packet in flight during registration process. In the case of MIP, the MN always uses its home address to send and receive packets, and it cannot contact the old FA (satellite) we can see there is a transmission stall of about $240 \mathrm{~ms}$, which represents the handover latency when using MIP. This handover latency is independent from the time spent in overlapping area of new and old satellite. In SeaHO-LEO, as soon as the old Satellite receives HR_REQUEST message it establishes a virtual communication path between the new satellite and MN by the help of ISL between it and new satellite. This process needs approximately $20 \mathrm{~ms}$. In our proposed method there is no need to send or receive HR_REQUEST and no need to wait as the $\mathrm{HM}$ itself initiates every handover. That is why the handover latency is very less in our proposed method.

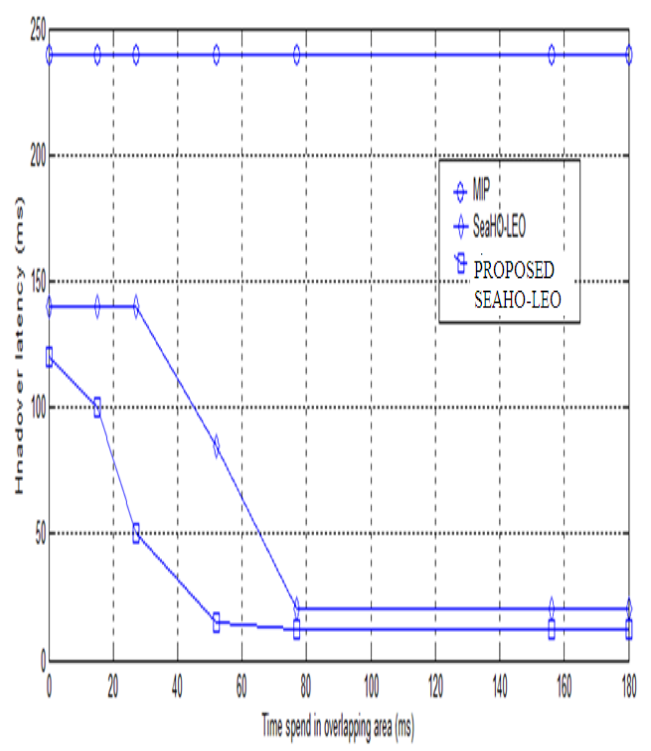

Figure 6: Handover latency

\section{CONCLUSION}

In this paper we have proposed a HANDOVER MANAGER concept to modify the handover technique of SeaHO-LEO satellite handover management where we have shown that our proposed method is more effective than the existing one by also showing theoretically as well as by simulation that it reduces handover latency, data loss, scanning time, cost and forced call termination probability. Our proposed handover method is also better as compare to the time delay and the binding updates as it itself compares the signal strength and chooses the next ip where the packets are to be forwarded it has reduced the extra binding updates and as the search is done when the previous data is being forwarded it also reduces the searching time after the first data sent. So comparing all the aspects our work is practically applicable to any areas. In future we will find how to improve the efficiency of HM. Also we have to find a specific algorithm under which it will select the appropriate next IP in the network where the data is to be sending next. We can also used different algorithm according to the signal strength of the MNs. As the scanning time is reduced so we must search for appropriate threshold level under which the handover procedures will be started.

\section{REFERENCES}

[1] S. L. Kota, P. A. Leppanen, and K. Pahlavan, Broadband SatelliteCommunications For Internet Access, Kluwer Academic Publishers, 2004.Jamalipour, "Satellites in IP networks," in Wiley Encyclopedia of

[2] Telecommunications, vol. 4, Wiley, 2002, pp. 21112122.

[3] L. Strand, "Linux mobile IPv6 HOWTO,” Apr. 2004.

[4] Satellite Mobility Pattern Scheme for Centrical and Seamless Handover Management in LEO SatelliteNetworksAys, eg"ul T"uys"uz and Fatih Alag"oz

[5] H. Uzunalioglu, I. F. Akyildiz, Y. Yesha, and W. Yen, "Footprint handoverRerouting protocol for low earth orbit satellite networks," Wireless Networks, vol. 5, no. 5, pp. 327-337, 1999

[6] Systems By Joydeep Banerjee D Sarddar, S.K. Saha, M.K. Naskar, T.Jana, U. Biswas

[7] J. T. Malinen and C.Williams, "Micromobility taxonomy," Internet Draft,IETF, Nov. 2001

[8] P. Bhagwat, C. Perkins, and S. Tripathi, "Network layer mobility: An architecture and survey," IEEE Pers. Commun., vol. 3, no. 3, pp. 54-64, June1996.

[9] A. T. Campbell, J. Gomez, S. Kim, Z. Turanyi, C.-Y. Wan, and A. Valko, Comparison of IP micro-mobility protocols," IEEE Wireless Commun. Mag., vol. 9, no. 1, Feb. 2002.

[10]H. Tsunoda, K. Ohta, N. Kato, and Y. Nemoto, "Supporting IP/LEO satellite networks by handoverindependent IP mobility management," IEEE J.Select. Areas Commun., vol. 22, no. 2, pp. 300-307, 2004.

[11]X. Zhang, J. G. Castellanos, and A. T. Campbell, "Design and performance of mobile IP paging," ACM Mobile Networks and Appl., vol. 7, no. 2, Mar.2002. 
[12] A. G. Valko, "Cellular IP-a new approach to Internet host mobility," ACMComputer Commun. Rev., Jan. 1999.

[13] C. Perkins, "IP mobility support," RFC 2002, IEFT, Oct. 1996, available at http://www.ietf.org/rfc/rfc2002.txt.

[14] K. Leung, D. Shell, W. Ivancic, D. Stewart, T. Bell, and B. Kachmar, "Application of mobile-IP to space and aeronautical networks," IEEE Aerosp. Electron. Syst. Mag., vol. 16, no. 12, pp. 13-18, Dec. 2001.

[15] M. Atiquzzaman, S. Fu, and W. Ivancic, "TraSH-SN: A transport layer seamless handoff scheme for space networks," in Proc. ESTC 2004, Palo Alto, CA, June 2004.

[16] Y. H. Kwon and D. K. Sung, "Analysis of handover characteristics in shadowed LEO satellite communication networks," Int. J. Satellite Commun.,vol. 19, no. 6, pp. 581-600, Nov. 2001.

[17] R. Droms, "Dynamic host configuration protocol (DHCP)," RFC 2131,IETF, Mar. 1997.

[18]R. Droms, "Stateless dynamic host configuration protocol (DHCP) service for IPv6," RFC 3736, IETF, Apr. 2004.

[19] S. Thomson and T. Narten, "IPv6 stateless address autoconfiguration,"RFC 2462, IETF, Dec. 1998.

[20] Minimization of Call Blocking Probability using Auxiliary Stations by D.sarddar,shubhajeet chatterjee,mandovi mukherjee,ijcaonline.org, International Journal of Computer Applications (0975 8887) Volume 25-No.7, July 2011

[21] Debabrata Sarddar, Soumya Das,Dipsikha Ganguly,Sougata Chakraborty,M.k.Naskar, A New Method for Fast and Low Cost Handover in Leo Satellites(International Journal of Computer Applications (0975 - 8887) Volume 37- No.7, January 2012)

http://www.ijcaonline.org/archives/volume37/number7/4 622-6631

[22] Debabrata Sarddar, Soumya Das,Dipsikha Ganguly,Sougata Chakraborty,M.k. Naskar,A New Method for Controlling Mobility Management Cost of PatHO- LEO Satellite and Mobile IP Network (International Journal of Computer Applications (0975 8887)Volume37-No.7,January2012

http://www.ijcaonline.org/archives/volume37/number7 /4621-6630

\section{AUTHORS PROFILE}

Debabrata Sarddar is currently pursuing his $\mathrm{PhD}$ at Jadavpur University. He completed his M.Tech in Computer Science \& Engineering from DAVV, Indore in 2006, and his B.Tech in Computer Science \& Engineering from Regional Engineering College, Durgapur in 2001. His research interest includes wireless and mobile communicationDipsikha Ganguly, currently pursuing B.Tech in Electronics \& Communication engineering at B.P.Poddar Institute of Management \& Technology under West Bengal University of Technology.
Her research interest includes wireless communication \& satellite communication

Soumya Das, son of Mr. Subrata Das and Mrs. Swapna Das, currently pursuing his B.Tech in Electronics \& Communication Engg. at Bengal Institute of Technology under West Bengal University of Technology. His research interest includes mobile communication \& satellite communication.

Suman Kumar Sikdar completed his B.Tech in CSE from Murshidabad College of Engineering \& Technology and M.Tech in Computer Science \& Engineering from Jadavpur University in 2011. His research interest includes wireless sensor networks and wireless communication systems.

Sougata Chakraborty is presently an employee of IBM (Kolkata). He has completed his B.Tech in IT from Murshidabad College of Engineering and Technology and M.Tech in Computer Science \& Engineering from Jadavpur University in 2011. His research interest includes wireless sensor networks and wireless communication systems.

Kunal Hui is presently an employee of Cognizant (Kolkata). $\mathrm{He}$ has completed his B.Tech in Computer Science \& Engineering from Murshidabad College of Engineering and Technology (2004-2008) and M.Tech in Computer Science \& Engineering from Haldia Institute of Technology (20092011). His research interest includes wireless sensor networks and wireless communication systemShabnam Bandyopadhyay is presently an employee of Cognizant (Kolkata). She has completed her B.Tech in Electronics and Communication Engineering from Meghnad Saha Institute of Technology and pursuing M.Tech degree in Computer Science \& Engineering at Jadavpur University Her research interest includes wireless sensor networks and wireless communication systems.

Kalyan Kumar Das completed his BE degree in Computer Science and Engineering from REC (NIT), Durgapur in 2002 and M.Tech. in Computer Technology from Jadavpur University in 2011. His research interest includes wireless sensor networks and wireless communication systems

Mrinal Kanti Naskar received his B.Tech. (Hons) and M.Tech degrees from E\&ECE Department, IIT Kharagpur, India in 1987 and 1989 respectively and Ph.D. from Jadavpur University, India in 2006.. He served as a faculty member in NIT, Jamshedpur and NIT, Durgapur during 1991-1996 and 1996-1999 respectively. Currently, he is a professor in the Department of Electronics and Tele-Communication Engineering, Jadavpur University, Kolkata, India where he is in charge of the Advanced Digital and Embedded Systems Lab. His research interests include ad-hoc networks, optical networks, wireless sensor networks, wireless and mobile networks and embedded systems. He is an author/co-author of the several published/accepted articles in WDM optical networking field that include "Adaptive Dynamic Wavelength Routing for WDM Optical Networks" [WOCN,2006], "A Heuristic Solution to SADM minimization for Static Traffic Grooming in WDM uni-directional Ring Networks" [Photonic Network Communication, 2006], "Genetic Evolutionary Approach for Static Traffic Grooming to SONET over WDM Optical Networks" [Computer Communication, Elsevier, 2007], and "Genetic Evolutionary Algorithm for Optimal Allocation of Wavelength Converters in WDM Optical Networks" [Photonic Network Communications, 2008]. Fast Handoff Implementation by using Curve Fitting Equation With Help of GPS 